\title{
Effective Passivation of Black Phosphorus under Ambient Conditions
}

\author{
Jongchan Yoon, Zonghoon Lee* \\ School of Materials Science and Engineering, Ulsan National Institute of Science and Technology (UNIST), UIsan 44919, Korea
}

*Correspondence to:

Lee $\mathrm{Z}$,

Tel: +82-52-217-2327

Fax: +82-52-217-2309

E-mail: zhlee@unist.ac.kr

Received August 31, 2017

Revised September 10, 2017

Accepted September 10, 2017
Two-dimensional (2D) materials have been studied widely owing to their outstanding properties since monolayer graphene was isolated in 2004. Especially, among 2D materials, phosphorene, a single atomic layer of black phosphorus (BP), has been highlighted for its electrical properties. This material can serve as a substitute for graphene, which has been revealed as a "semi-metal", in next-generation semiconductors. However, few-layer $\mathrm{BP}$ is prone to degradation under ambient conditions owing to its reactivity with oxygen and water, which results in the condensation of water droplets on the surface of the BP flakes. This causes charge transfer from the phosphorus atom to oxygen, resulting in the formation of phosphoric acid (oxide) and degrades the various properties of BP. Therefore, it is necessary to find passivation methods to prevent BP flakes from being degraded under ambient conditions. This review article deals with recent studies on passivation methods for BP and their performance against oxygen and water, effects on the electrical properties of $\mathrm{BP}$, and the extent to how they protect $\mathrm{BP}$.

Key Words: Two-dimensional materials, Black phosphorus, Photooxidation, Degradation, Passivation

\section{INTRODUCTION}

Since the discovery of an exfoliated monolayer graphene in 2004, two-dimensional (2D) materials have attracted considerable attention for use in future technologies, and have been investigated extensively owing to their intrinsic potentials, such as excellent thermo-mechanical properties, and charge transport properties, which would help surpass obstacles hampering their application as semiconductor devices (Geim \& Novoselov, 2007). For example, graphene, a single atomic layer of graphite, showed potential as a nextgeneration semiconductor material owing to its unique mechanical, optical, and electrical properties. However, a symmetrical dispersion relation at the Dirac point, which indicates graphene is a semi-metal, limits its application in the semiconductor industry (Lee et al., 2015).

Phosphorene (Fig. 1), a monolayer of black phosphorus (BP), has recently attracted attention owing to its intriguing anisotropic opto-electronic properties. Particularly, owing to its outstanding properties such as thickness-dependent direct band gap $(0.3 \sim 2.0 \mathrm{eV}$ ), high carrier mobility (up to $\left.1,000 \mathrm{~cm}^{2} /(\mathrm{Vs})\right)$, and current on/off ratio, BP is an upcoming candidate for next generation nanoscale electronic device (Kim et al., 2015).

However, few-layer BP is chemically unstable and tends to be degraded immediately under ambient conditions owing to oxidation of the surface of BP flakes. An experiment for uncovering this mechanism of degradation was conducted by Favron et al. (2015). Fig. 2A and B show atomic force microscopy (AFM) images of the BP flakes exfoliated using an adapted Scotch-tape exfoliation method. Right after exfoliation, as shown in Fig. 2A, small bumps can be seen on the surface, and after a few days under ambient conditions, as in Fig 2B, large droplets are formed owing to continuous degradation.

Raman spectra of 10-layer of BP are shown in Fig. 2C, and

(a) This is an open-access article distributed under the terms of the Creative Commons Attribution Non-Commercial License (http://creativecommons.org/licenses/by-nc/4.0) which permits unrestricted noncommercial use, distribution, and reproduction in any medium, provided the original work is properly cited.

Copyrights @ 2017 by Korean Society of Microscopy 

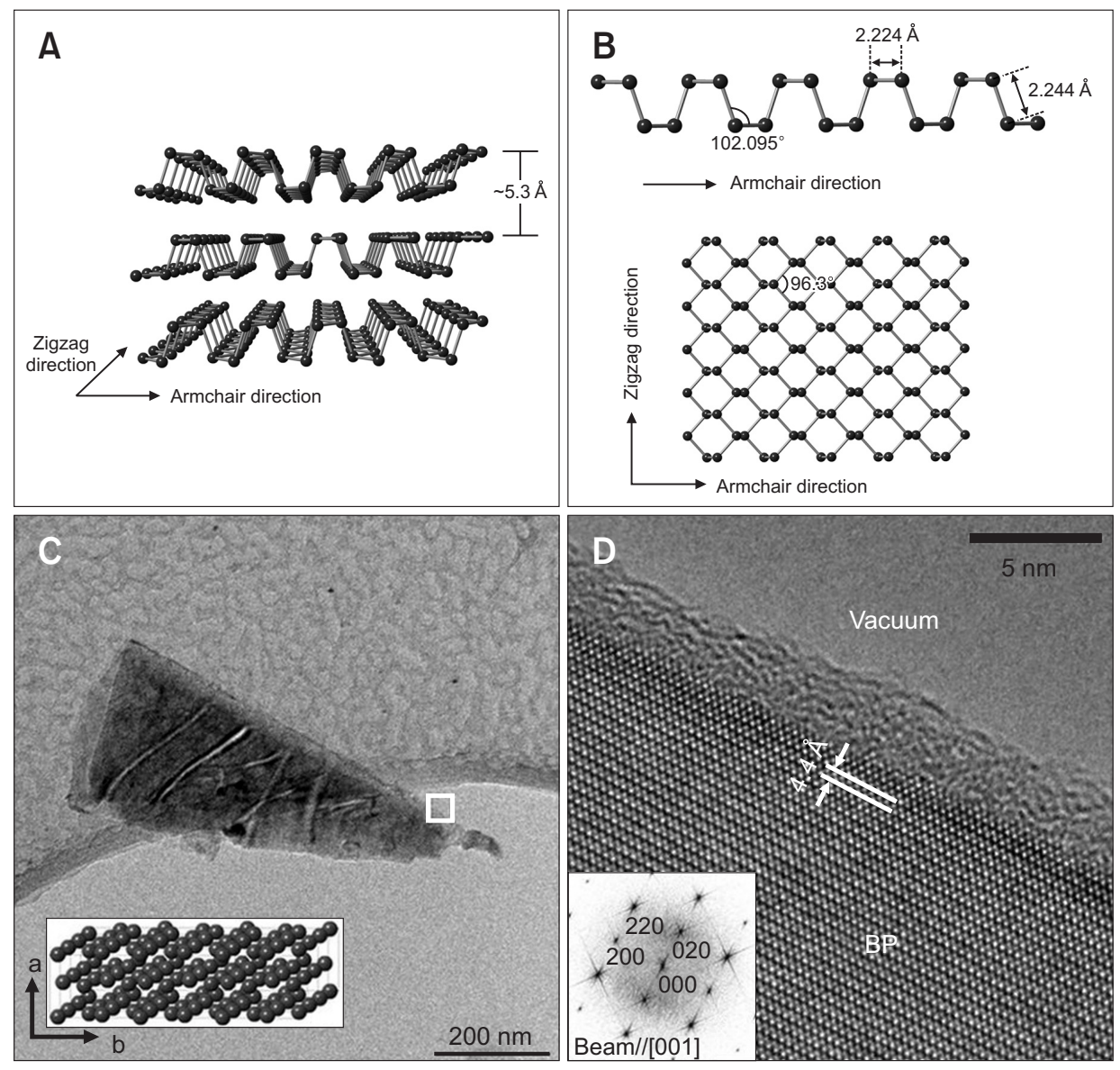

Fig. 1. (A) Atomic structure of few-layer black phosphorus, (B) a monolayer of black phosphorus (phosphorene), (C) bright-field transmission electron microscopy (TEM) image of black phosphorus (BP) flakes prepared by liquid phase exfoliation (inset: atomic model of BP), (D) high-resolution TEM image of BP (inset: fast Fourier transform). (A, B) Reprinted from Du et al. (2015) (J. Mater. Chem. C 3, 87608775), with permission from Royal Society of Chemistry. (C, D) Reprinted from Seo et al. (2016) (Sci. Rep. 6, 23736), with permission from Nature.

they were measured at 24, 48, 96, and $120 \mathrm{~min}$ after exposure to ambient conditions with a laser fluence of $2 \times 10^{4} \mathrm{~W} \mathrm{~cm}^{-2}$ at a wavelength of $532 \mathrm{~nm}$. The Raman modes corresponding to $361 \mathrm{~cm}^{-1}, 438 \mathrm{~cm}^{-1}$, and $466 \mathrm{~cm}^{-1}$ are assigned $\mathrm{A}_{\mathrm{g}}{ }^{1}, \mathrm{~B}_{2 \mathrm{~g}}$, and $\mathrm{A}_{\mathrm{g}}{ }^{2}$, respectively. Although there is no significant change in the positions and the widths of the peaks, the decreased Raman intensities are attributed directly to sample degradation, indicating that the structural properties of BP are disrupted by this degradation.

To clarify the occurrence of degradation, Favron et al. (2015) exposed the BP samples to different conditions. Raman spectra of the BP samples were recorded under different conditions: air, mixture of $\mathrm{O}_{2}$ and $\mathrm{H}_{2} \mathrm{O}$, vacuum, and in the presence of laser, which corresponds to the wavelength of visible light (Fig. 2D and E). The normalized Raman intensities of only $\mathrm{O}_{2}$ and only $\mathrm{H}_{2} \mathrm{O}$ in Fig. $2 \mathrm{E}$ remain almost constant, meaning degradation did not occur when BP was exposed to either oxygen or water. According to the changes in the Raman intensities of the mixture of $\mathrm{O}_{2}$ and $\mathrm{H}_{2} \mathrm{O}$, and air, it is certain that degradation occurs in environments with both oxygen and water (or air). Moreover, the light-induced oxidation mechanism of BP (photooxidation) revealed by Favron et al. (2015) suggests that oxidation occurs due to transfer of photon-induced electrons from phosphorus atoms to aqueous oxygen molecules, which coincides with the water condensation. As a result of the photooxidation, an ambipolar BP turns into a p-type doped BP. Therefore, light, oxygen, and water can be regarded as the primary factors for BP degradation.

It has been reported that the transfer characteristic of a pristine BP field-effect transistor (FET) shows a right-shifted threshold voltage, which might be considered that a pristine $\mathrm{BP}$ is a p-type semiconductor. In fact, however, a pristine BP shows ambipolar behavior; instead, once a BP is exposed to air, an immediate photooxidation occurs, which leads the BP to a p-type doped semiconductor. In the same manner, the threshold voltage shifts toward the right as the exposure time increases, eventually, breaks down after $56 \mathrm{~h}$. Once water condensation occurs on the BP flakes, charge transfer from phosphorus to aqueous oxygen molecule take place, making BP a p-doped semiconductor, which corresponds to the threshold voltage shift toward the right (Wood et al., 2014).

Thus, the development of new passivation methods to protect BP flakes from degradation it has become have been raised as a primary research topic in the use of BP, and various reliable passivation methods have been suggested. 
A

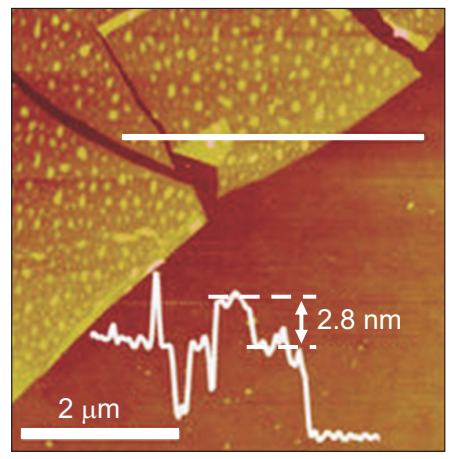

B

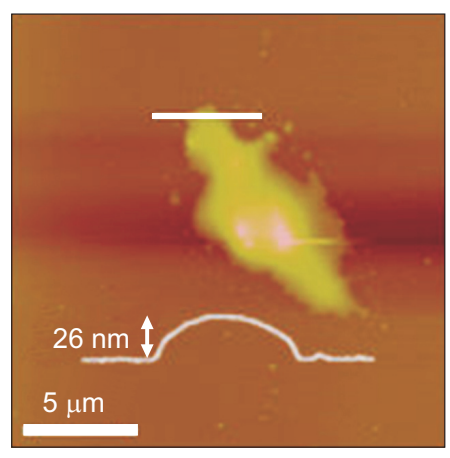

\section{D}

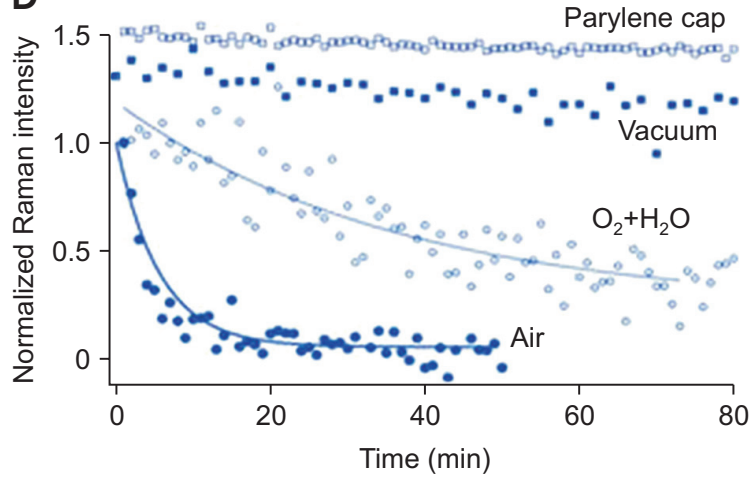

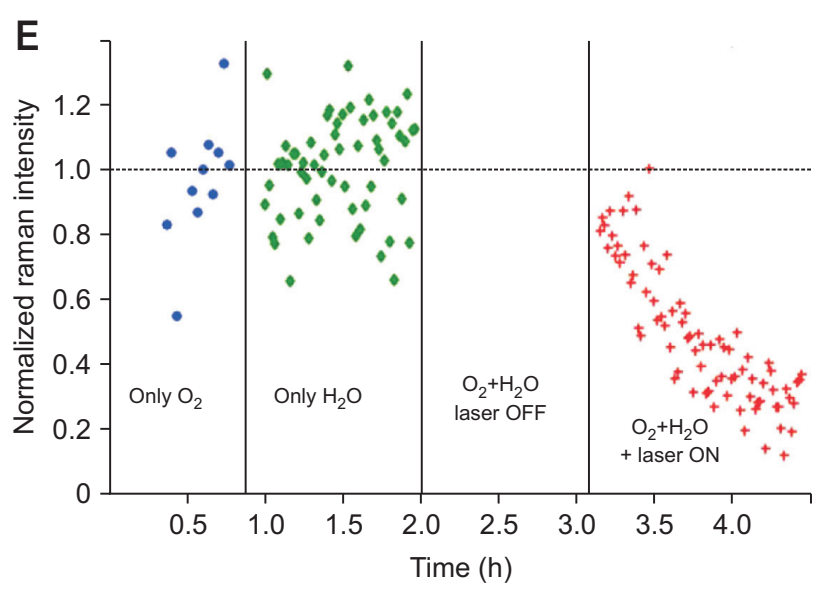

Fig. 2. Atomic force microscopy (AFM) scans of black phosphorus (BP) flake under ambient condition, immediately after exfoliation (A) and after a few days under ambient conditions (B) (insets: height contours of AFM across the white line). (C) Raman spectra of a $\sim 5 \mathrm{~nm}$ thick flake measured under ambient conditions at $24,48,96$, and $120 \mathrm{~min}$ after exfoliation (black arrow shows passage of time from blue to purple). (D) Normalized Raman intensities of $\mathrm{A}_{\mathrm{g}}^{2}$ versus exposure time under different conditions: air, vacuum, mixture of $\mathrm{O}_{2}$ and $\mathrm{H}_{2} \mathrm{O}$, and encapsulated with a $300 \mathrm{~nm}$-thick of parylene cap. (E) Development of $\mathrm{A}_{\mathrm{g}}^{2}$-integrated Raman intensity of few-layer BP under different conditions (Laser wavelength: $532 \mathrm{~nm}$, fluence: $\left.200 \mu \mathrm{W} \mu \mathrm{m}^{-2}\right)$. Reprinted from Favron et al. (2015) (Nat. Mater. 14, 826832), with permission from Nature.
In this paper, three passivation methods focusing on preventing the degradation of BP are introduced. Roughly, there are two types of passivation methods: a direct transfer of inert 2D materials (graphene and hexagonal boron nitride [hBN] onto BP flakes by van der Waals bonds, and formation of atomic layer deposition (ALD)-derived $\mathrm{AlO}_{\mathrm{x}}$ onto the $\mathrm{BP}$ surface and generation of phosphorus-carbon bonds by using organic solvents. By observing the progress of degradation using microscopes and spectroscopes, we can easily figure out whether the passivation methods are effective.

\section{PASSIVATION METHOD}

\section{Chemical Passivation}

\section{Covalent functionalization and passivation via aryl diazonium}

Diazonium compounds are a group of organic compounds consisting of a functional group $\mathrm{R}-\mathrm{N}_{2}{ }^{+} \mathrm{X}^{-}$where $\mathrm{R}$ is an organic group, such as an alkyl or an aryl, and $\mathrm{X}$ is an organic or inorganic anion. Especially, in this experiment, Ryder et al. (2016) used aryl diazonium compounds, where $\mathrm{R}$ is an aryl group, which is any functional group derived from an aromatic ring. Aryl diazonium compounds, which makes 
A

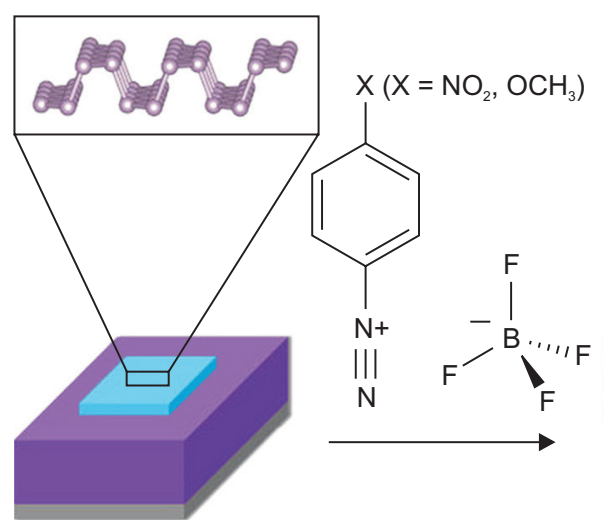

B
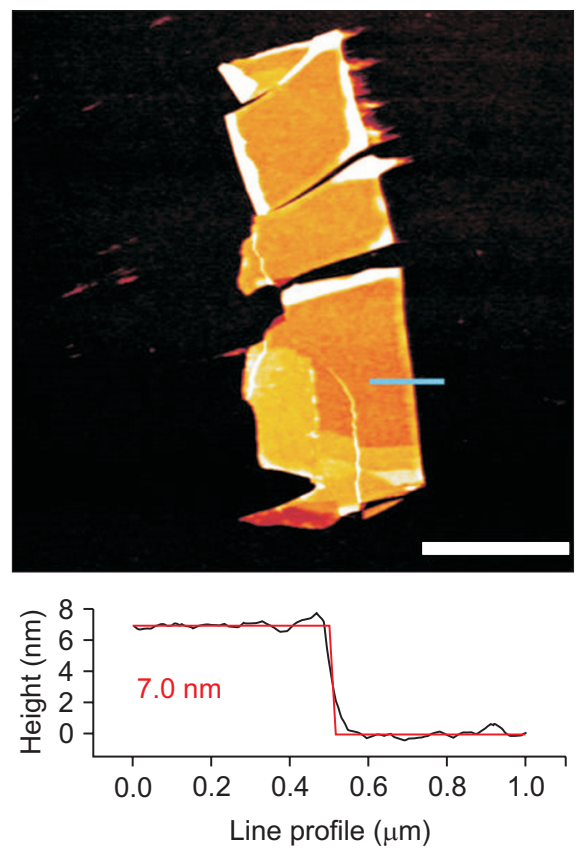
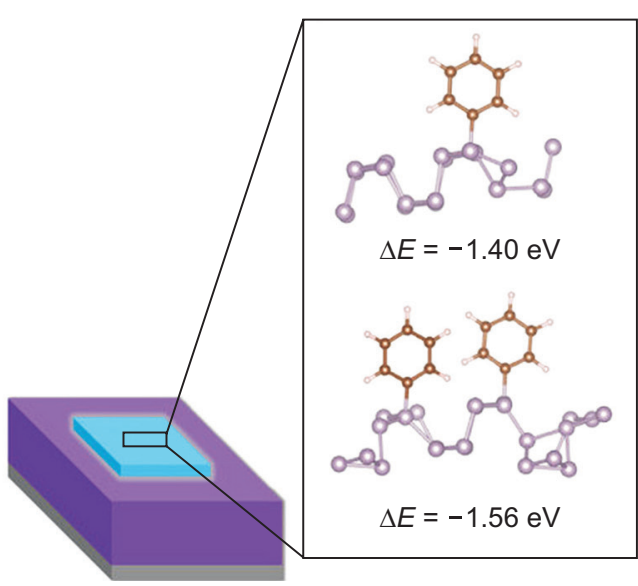

C
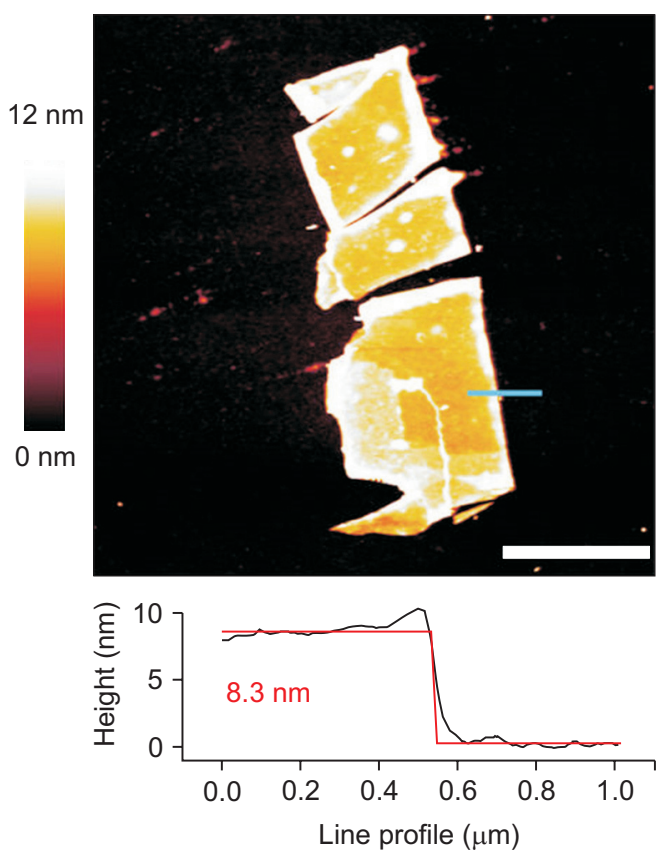

Fig. 3. Functionalization of aryl diazonium on black phosphorus (BP). (A) Functionalization scheme of aryl diazonium tetrafluoroborate and fewlayer BP (light blue) mechanically exfoliated onto a $\mathrm{Si}$ (grey) $/ \mathrm{SiO}_{2}$ (purple) substrate. The left panel shows the atomic structure of pristine $\mathrm{BP}$ and the right panel shows the atomic structures of aryl groups on BP based on the density functional theory calculations. (B) Atomic force microscopy (AFM) profiles of BP flakes before functionalization (top) followed by height contour measured along blue line (bottom). (C) AFM profiles of same flakes after $30 \mathrm{~min}$ of modification to $10 \mathrm{mM} 4$-nitroenzenediazonium, followed by height contour measured along blue line (bottom), which indicates increased flakes height due to functionalization of aryl groups. Reprinted from Ryder et al. (2016) (Nat. Chem. 8, 597-602), with permission from Nature.

covalent bonds to carbon, are known for modification of opto-electronic properties of materials, such as enhancing the photoluminescence emission of CNTs and producing a bandgap in graphene (Piao et al., 2013).

Ryder et al. (2016) chemically modified few-layer BP mechanically exfoliated onto $\mathrm{Si} / \mathrm{SiO}_{2}$ substrate, with 4-nitroenzene-diazonium (4-NBD) and 4-methoxybenzenediazonium (4-MBD). Functionalization, which leads to the formation of firm P-C bonds between phosphorus atoms and aryl diazonium radicals, was conducted by dipping the samples into aryl diazonium salt solutions (Fig. 3A) After functionalization, the samples were rinsed with acetonitrile to prevent physisorption of other molecules.

Ryder et al. (2016) measured the thickness changes of the BP flakes by using AFM, and Fig. 3B and C show AFM images of the sample before and after modification for $30 \mathrm{~min}$ in 10 $\mathrm{mM}$ of 4-NBD solution. The height increases from about 7.0 $\mathrm{nm}$ to about $8.3 \mathrm{~nm}$, which is consistent with surface chemical modification $(\sim 1.3 \mathrm{~nm})$.

Ryder et al. (2016) obtained Raman spectra of BP flakes of $10 \sim 15 \mathrm{~nm}$ in thickness to check whether there is further progress of $\mathrm{BP}$ functionalization. The $\mathrm{A}_{\mathrm{g}}^{1}$ mode of $\mathrm{BP}$ at 361 $\mathrm{cm}^{-1}$ corresponds to out-of-plane atomic displacements (Sugai \& Shirotani, 1985).

The intensity of the $\mathrm{A}_{\mathrm{g}}^{1}$ mode decreased with increasing time for both 4-NBD and 4-MBD (Fig. 4). The integrated normalized peak intensities are plotted in Fig. 2C, where it is obvious that upon functionalization with $4-\mathrm{NBD}$, the $\mathrm{A}_{\mathrm{g}}^{1}$ diminishes more rapidly than that when functionalized with 4-MBD. The weakening of the Raman modes of the BP of 

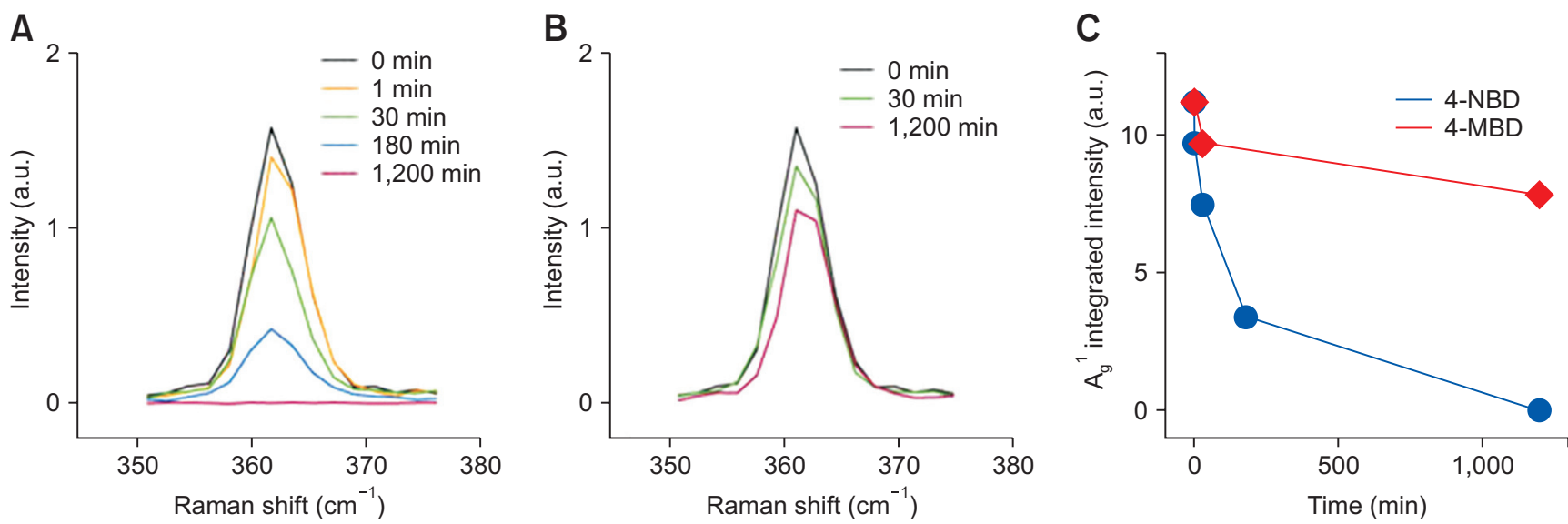

Fig. 4. (A) The black phosphorus (BP) $A^{1}{ }_{g}$ mode weakens with increasing immersing time in 4-nitroenzene-diazonium (4-NBD). (B) The $A_{g}^{1}$ mode shows comparable aspect for 4-methoxybenzene-diazonium (4-MBD) with a reduced rate. (C) Integrated $\mathrm{A}_{\mathrm{g}}^{1}$ intensities from (A) and (B) describing the rates of charge transfer (functionalization) from BP functionalized with 4-NBD and with 4-MBD. Reprinted from Ryder et al. (2016) (Nat. Chem. 8, 597-602), with permission from Nature.
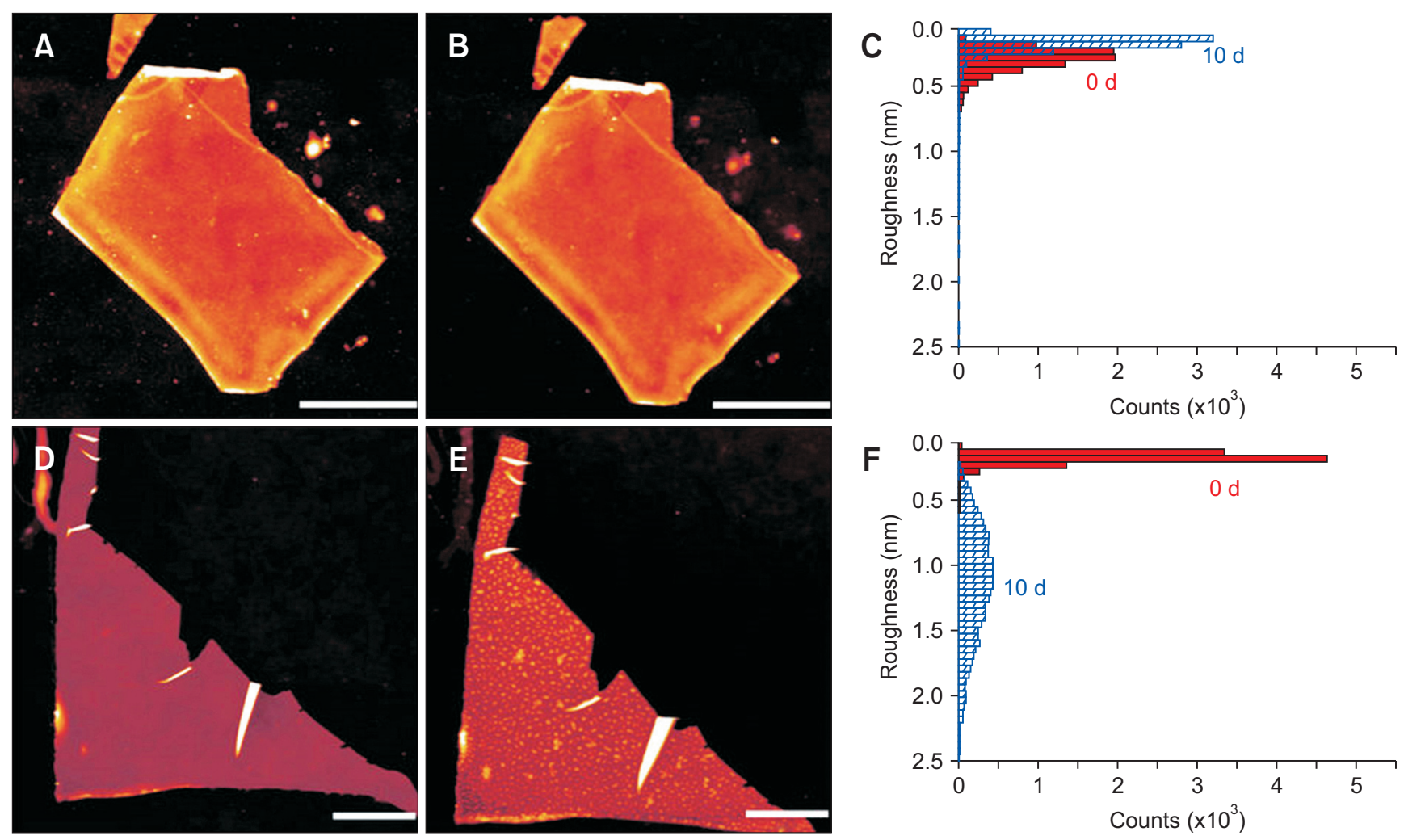

Fig. 5. Atomic force microscopy (AFM) micrograph of chemically passivated black phosphorus (BP) morphology. (A) BP flake right after functionalization with $10 \mathrm{mM}$ of 4-nitroenzene-diazonium (4-NBD) for $30 \mathrm{~min}$. (B) Same flakes as those in (A) after 10 days under ambient conditions. (C) Histograms of surface roughness of the flakes in (A) and (B). (D) Pristine BP flakes immediately after exfoliation. (E) The same flakes as in (C) after 10 days of exposure to ambient conditions. (F) Histograms of surface roughness of flakes in (D) and (E). Reprinted from Ryder et al. (2016) (Nat. Chem. 8, 597-602), with permission from Nature.

the functionalized samples demonstrates that intralayer $\mathrm{P}$ bonds are disrupted, which leads to the conclusion that the formation of covalent bonds of aryl groups produces BP lattice distortion.

Fig. 5 shows the AFM morphology results of pristine BP and chemically modified BP flakes, which exhibit enhanced 

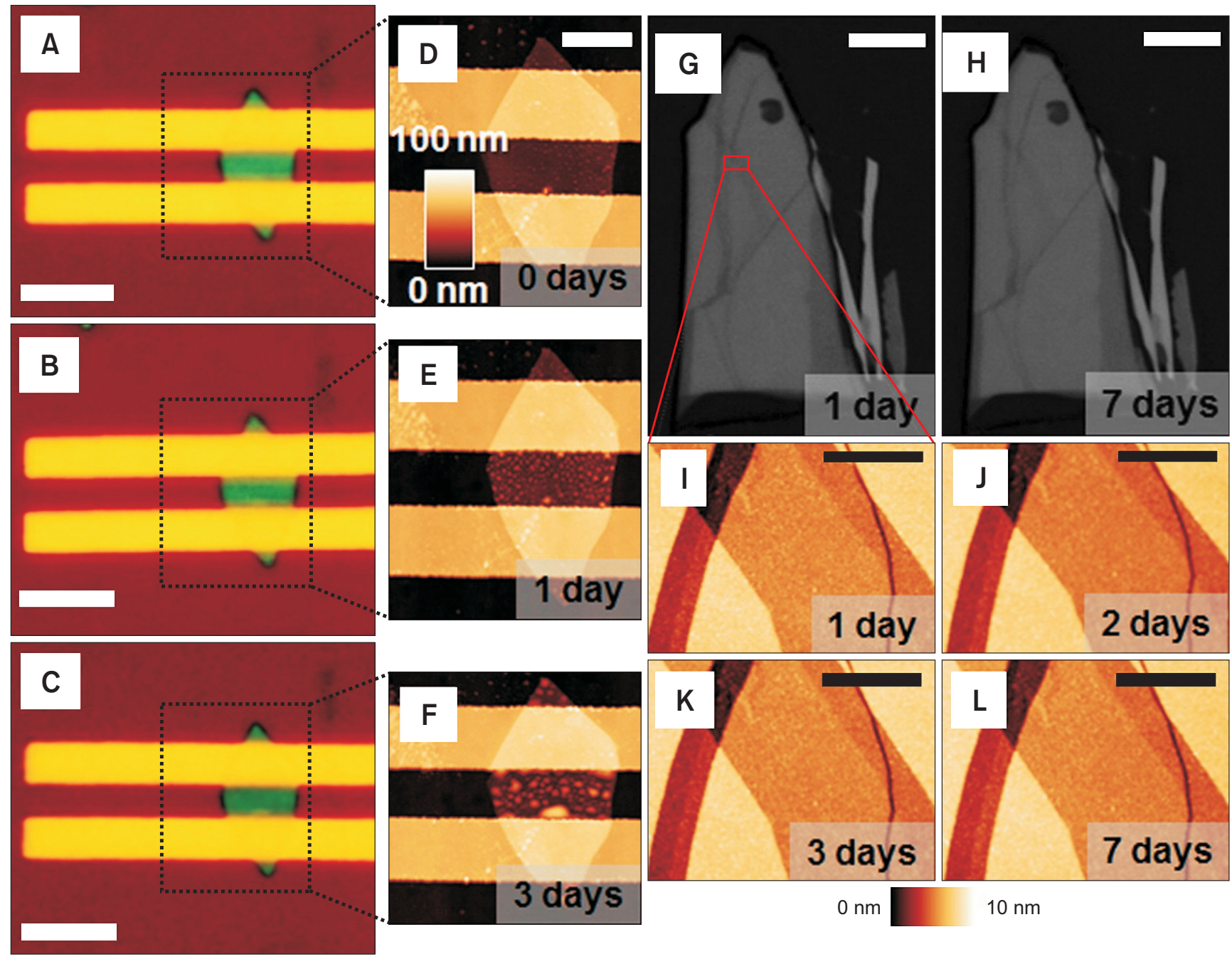

$0 \mathrm{~nm}$

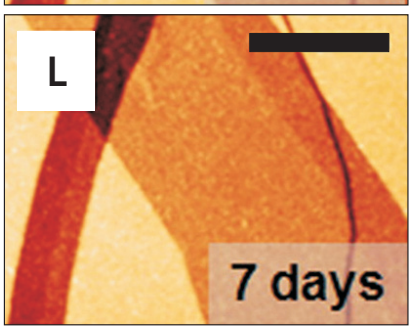

$10 \mathrm{~nm}$

Fig. 6. Passivation of exfoliated black phosphorus (BP) flake $(\sim 8.9 \mathrm{~nm})$ on $\mathrm{SiO}_{2}$ by atomic layer deposition-derived $\mathrm{AlO}_{\mathrm{x}}$. (A-C) show visible light microscope (VLM) images of an unpassivated BP field-effect transistor (FET) after 0 day, 1 day, and 3 days, respectively. Corresponding atomic force microscopy (AFM) height images of the boxed region after 0 day, 1 day, and 3 days (D-F) (scale bar $\sim 5 \mu \mathrm{m}$ ). VML images of $\mathrm{AlO}_{\mathrm{x}}$ passivated BP flakes $(\sim 92.7 \mathrm{~nm})$ on SiO${ }_{2}$ after $(\mathrm{G})$ 1 day and (H) 7 days under ambient conditions. No degradation was observed. (I-L) are AFM height images for passivated BP in (G) after 1 day, 2 days, 3 days, and 7 days under ambient conditions, respectively. No degradation occurs regardless of exposure time. Reprinted from Wood et al. (2014) (Nano Lett. 14, 6964-6970), with permission from American Chemistry Society.

chemical stability against ambient exposure, showing no discernible evidence of oxidation even after 10 days (Fig. 5AC). By contrast, pristine BP turns into a surface full of bubbles after the same duration of ambient exposure (Fig. 5D-F).

Moreover, after fabricating FET-devices using functionalized $\mathrm{BP}$, they measured the transfer characteristics of each devices in terms of functionalization time and observed different rates of the p-type doping effect for functionalization with 4-NBD and 4-MBD in addition to the p-type doping by an inevitable contact with aqueous oxygen molecules. The transfer curves (I-V characteristics) of each functionalized BP FET show that the doping rate of 4-NBD is much faster than that of 4-MBD, which corresponds to the result shown in the Raman spectra in Fig. 4.
In summary, by functionalizing $\mathrm{BP}$ with aryl diazonium compounds (4-NBD, 4-MBD), few-layer BP was passivated chemically, and showed little degradation according to the AFM measurements owing to the formation of P-C bonds which hinders charge transfer from phosphorus atoms to aqueous oxygen molecules; instead, charge transfer from phosphorus atoms to carbon atoms in the aryl diazonium radicals leads to p-type doping of $\mathrm{BP}$, thus enhancing the transfer characteristics of BP devices.

\section{Atomic layer deposition passivation}

ALD is a thin film deposition technique based on the serial use of chemical precursors. With typical ALD method, using trimethylaluminum and $\mathrm{H}_{2} \mathrm{O}$ as the precursors, Wood et al. 

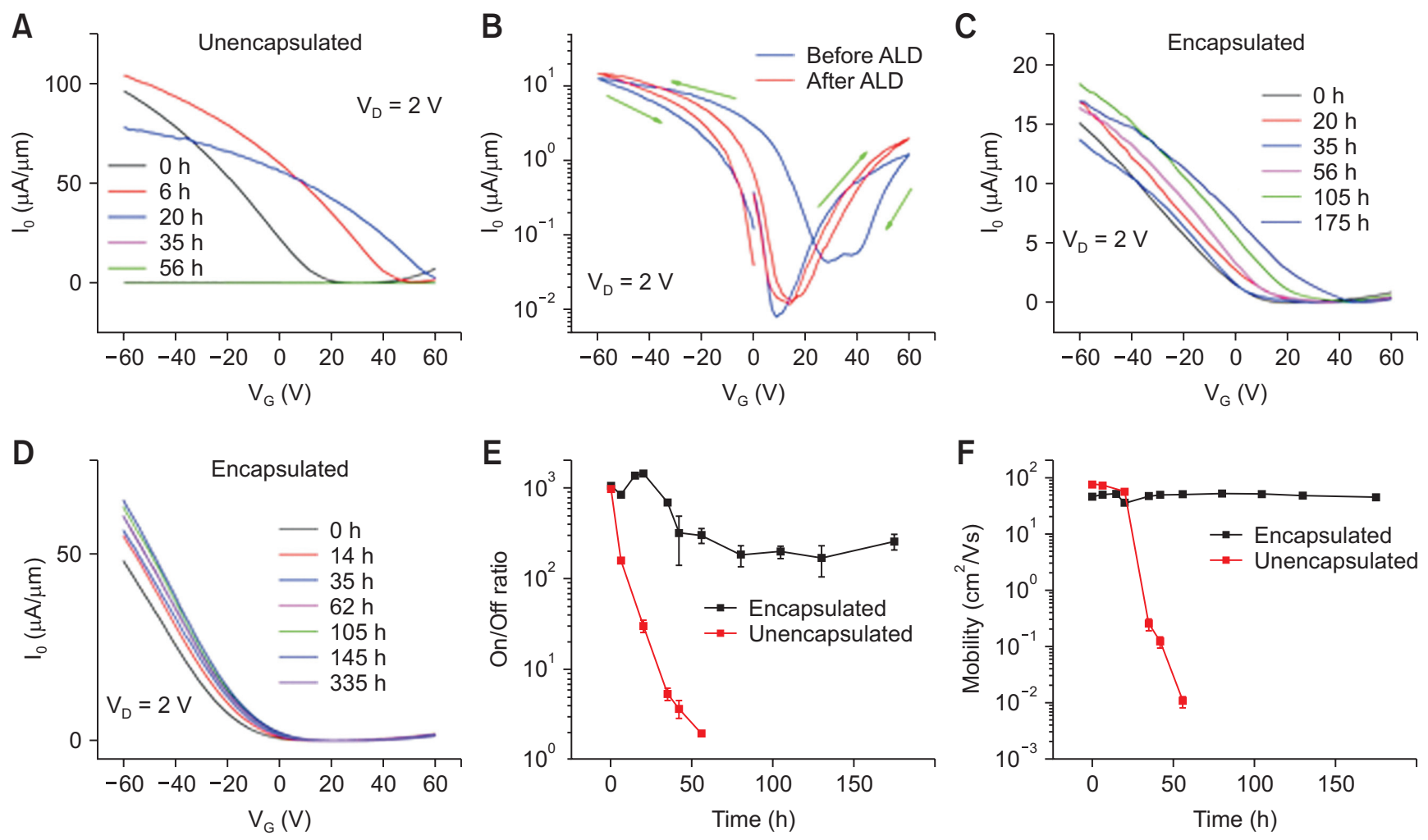

Fig. 7. Time-dependent characteristics of few-layer black phosphorus field-effect transistor (BP FET) device. (A) I- $V_{g}$ curves of unpassivated BP FET, as a function of exposure time under the ambient condition. (B) I- $V_{g}$ curves of BP FET, before and immediately after passivation. (C) I- $V_{g}$ curves of $\sim 30 \mathrm{~nm}$-thick atomic layer deposition (ALD)-derived $\mathrm{AlO}_{\mathrm{x}}$ passivated $\mathrm{BP} \mathrm{FET}$ with $\mathrm{Ti} / \mathrm{Au}$ contacts as a function of exposure time under ambient condition. (D) $\mathrm{I}-\mathrm{V}_{\mathrm{g}} \mathrm{curves}$ of $\sim 30 \mathrm{~nm}$-thick ALD-derived $\mathrm{AlO}_{\mathrm{x}}$ passivated BP FET with $\mathrm{Ni} / \mathrm{Au}$ contacts as a function of exposure time under ambient conditions. (E) On/Off current ratio and (F) hole mobility of passivated and unpassivated Ti/Au BP FET versus exposure time under ambient conditions. Reprinted from Wood et al. (2014) (Nano Lett. 14, 6964-6970), with permission from American Chemistry Society.

(2014) conducted BP passivation directly with an aluminum oxide layer $(\sim 3 \mathrm{~nm})$. By forming strong $\mathrm{OH}$ bonds with phosphorus atoms and an inert $\mathrm{AlO}_{\mathrm{x}}$ passivation layer onto the BP surface, there was no water condensation and charge transfer with aqueous oxygen (Ritala et al., 2000). The BP FET device was fabricated directly with $\mathrm{AlO}_{\mathrm{x}}$, and it exhibited longterm reliability against ambient condition, showing constant transport properties.

Fig. 6 shows the degradation of BP FET under ambient conditions. Fig. 6A-C show optical images of the unpassivated BP FET, and with the passage of time, we can see the color changes of the BP flakes from light green to dark green, which is a common occurrence in BP undergoing degradation. The corresponding AFM height images are shown in Fig. 6D-F, which show the bubbles on the surface of the $\mathrm{BP}$, which is the evidence of the degradation.

By contrast, in Fig. 6G-L, visible light microscope (VLM) images and AFM height images of the $\mathrm{AlO}_{\mathrm{x}}$ are shown. Fig. $6 \mathrm{G}$ and $\mathrm{H}$ show VLM images of an $\mathrm{AlO}_{\mathrm{x}}$-passivated BP flake after 1 day and 7 days of exposure to ambient condition; in these pictures, there is no evidence if degradation, and the
AFM height images show no degradation either.

To reveal the passivation effects of ALD-derived $\mathrm{AlO}_{\mathrm{x}}$ on FET device characteristics, BP devices were fabricated with $\mathrm{Ti} / \mathrm{Au}$ electrodes. The transfer curve of an as-fabricated BP FET is shown in Fig. 7A, and the initial on/off current ratio is $>10^{3}$ with a hole mobility of $74 \mathrm{~cm}^{2} /(\mathrm{Vs})$. After exposure to ambient conditions for $6 \mathrm{~h}, 20 \mathrm{~h}, 35 \mathrm{~h}$, and 56 $\mathrm{h}$, as the exposure time increases, the transfer curve shifts considerably, with the threshold voltage increasing by $22 \mathrm{~V}$, which corresponds to p-type doping of BP with oxidization, as indicated in previous studies. Eventually, after $56 \mathrm{~h}$, the BP FET device shows no I-V characteristics, which means the BP degraded completely into $\mathrm{PO}_{\mathrm{x}}$.

Fig. 7B shows the I-V characteristics of the BP FET before and after passivation. The on/off current ratio increases from 285 to over $10^{3}$. The passivated BP FET device is then placed under the same conditions as those in case of the unpassivated BP FET device. With the passage of time, there is a slight shift in the threshold voltage, but device performance remains unchanged (Fig. 7C). The performance was better when electrodes with $\mathrm{Ni} / \mathrm{Au}$ contacts were used (Fig. 7D) owing to 


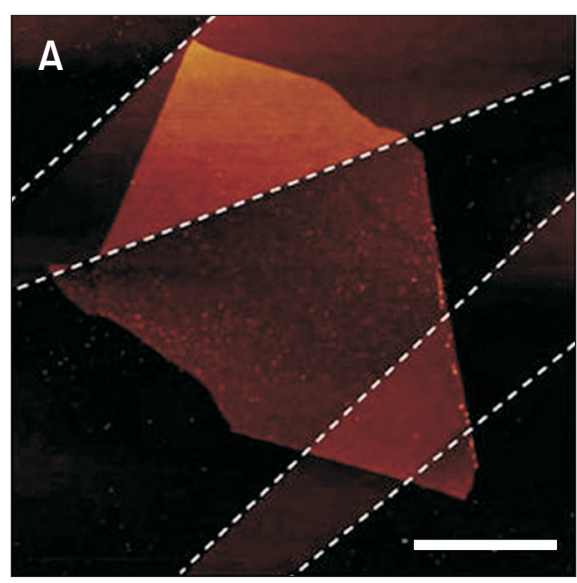

$0 \mathrm{~nm}$
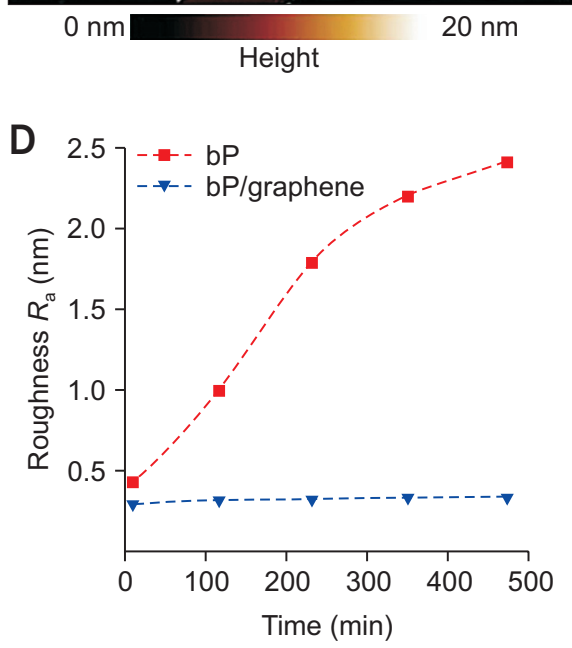

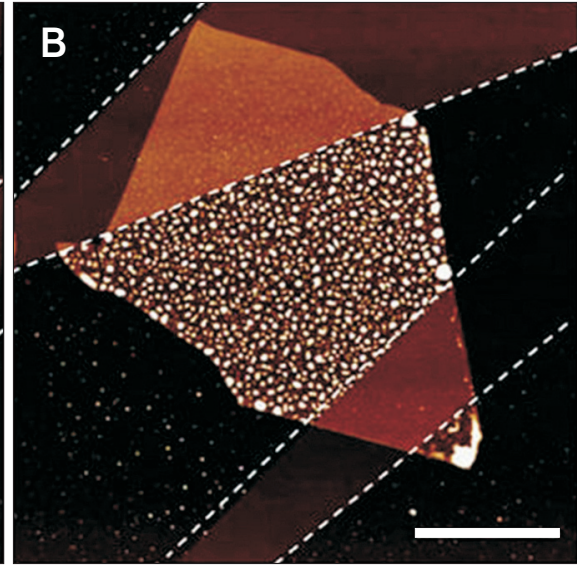

$0 \mathrm{~nm}$

E

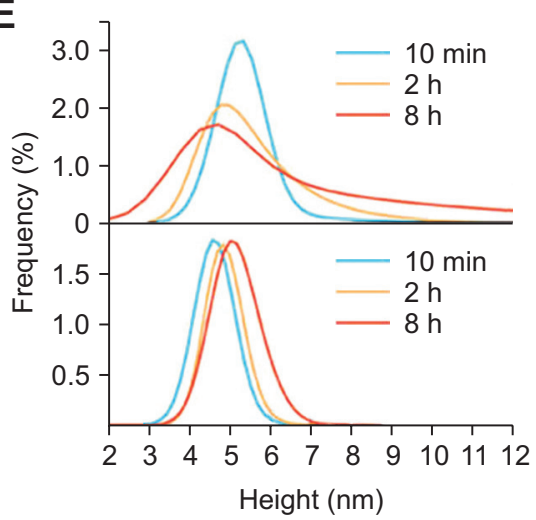

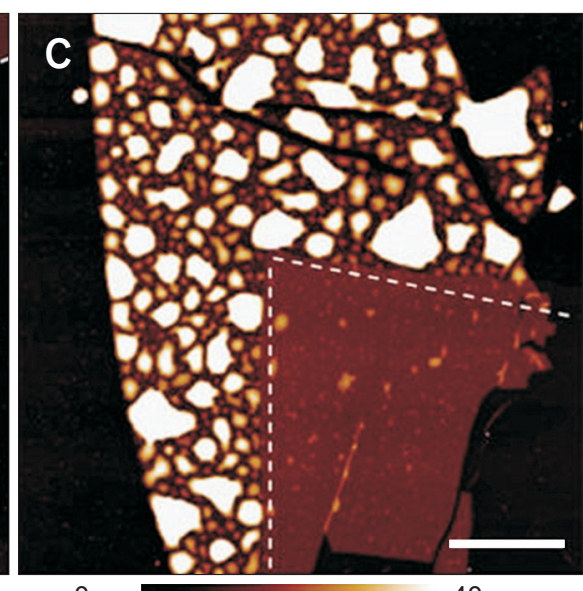

$0 \mathrm{~nm}$

$$
\text { Height }
$$

$\mathbf{F}$

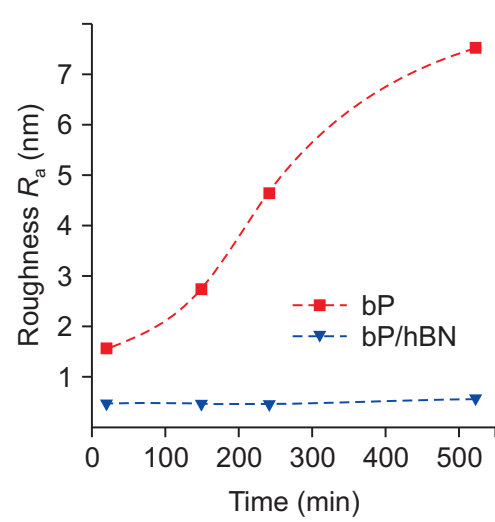

Fig. 8. Atomic force microscopy (AFM) measurements of passivated and unpassivated BP flakes under ambient condition (A), (B) AFM morphology of $5 \mathrm{~nm}$-thick black phosphorus (BP) crystal passivated partially with graphene. The images were taken after $10 \mathrm{~min}$ and $24 \mathrm{~h}$ exposure under ambient condition, respectively. (C) AFM morphology of $10 \mathrm{~nm}$-thick BP crystal passivated partially with hexagonal boron nitride (hBN) after 5 days under the ambient condition. (D) Roughness versus exposure times under ambient conditions for graphene/BP samples in (A) and (B). (E) Height profiles of unpassivated BP surface (top) and passivated BP surface (bottom) with different exposure times under the ambient condition. (F) Roughness versus exposure time under ambient condition for hBN/BP sample from (C). Reprinted from Doganov et al. (2015) (Nat. Commun. 6, 6647), with permission from Nature.

little shift of threshold voltage. Even, after 1week of ambient exposure, the passivated BP FET continued to function with almost constant hole mobility and slightly lowered on/off ratio (Fig. 7E and F).

In summary, the ALD-derived $\mathrm{AlO}_{\mathrm{x}}$ thin film effectively passivated few-layer $\mathrm{BP}$, preserving the surface roughness, on/ off ratio, and high carrier mobility of the BP FET.

\section{Physical Passivation}

\section{Graphene/hBN dry transfer passivation}

Among 2D materials, Graphene and hBN have outstanding mechanical properties, especially thermal stability, chemical inertness, and electrical properties: Graphene is a semimetallic material thath exhibits high conductivity, carrier mobility without electronic band gap. By contrast, $\mathrm{hBN}$ is a representative insulator that has large band gap, which is highlighted as a passivation layer for FET devices (Novoselov et al., 2005; Watanabe et al., 2004).

Doganov et al. (2015) conducted passivation through "dry transfer" with graphene and hBN onto few-layer BP prepared in $\mathrm{Ar}$ gas atmosphere to prevents degradation of pristine BP. They exfoliated either graphene or hBN onto $\mathrm{SiO}_{2} / \mathrm{Si}$ substrates coated with polymethylglutarimide and polymethylmethacrylate (PMMA) to help release and support graphene and hBN. After finding proper flakes, polymethylglutarimide was developed and the graphene (or hBN) held by the PMMA layer was then placed in the glovebox. To ensure perfect transfer, by using a micromanipulator in the glovebox, they lowered the material (Graphene, hBN) held by the PMMA polymer onto the exfoliated BP and then removed the PMMA layer (Tan et al., 2014). 


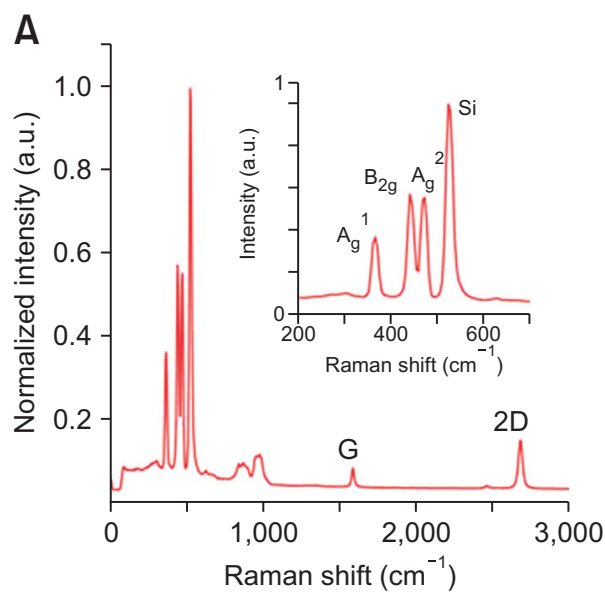

B

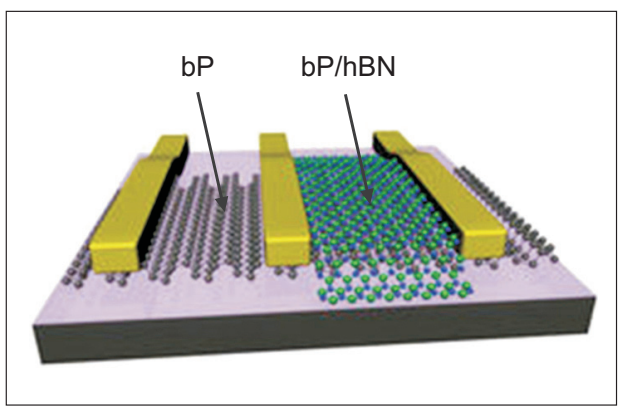

C

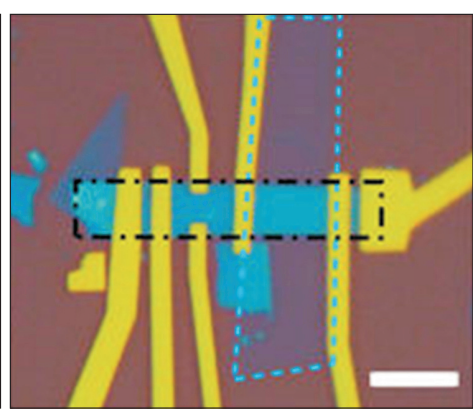

D
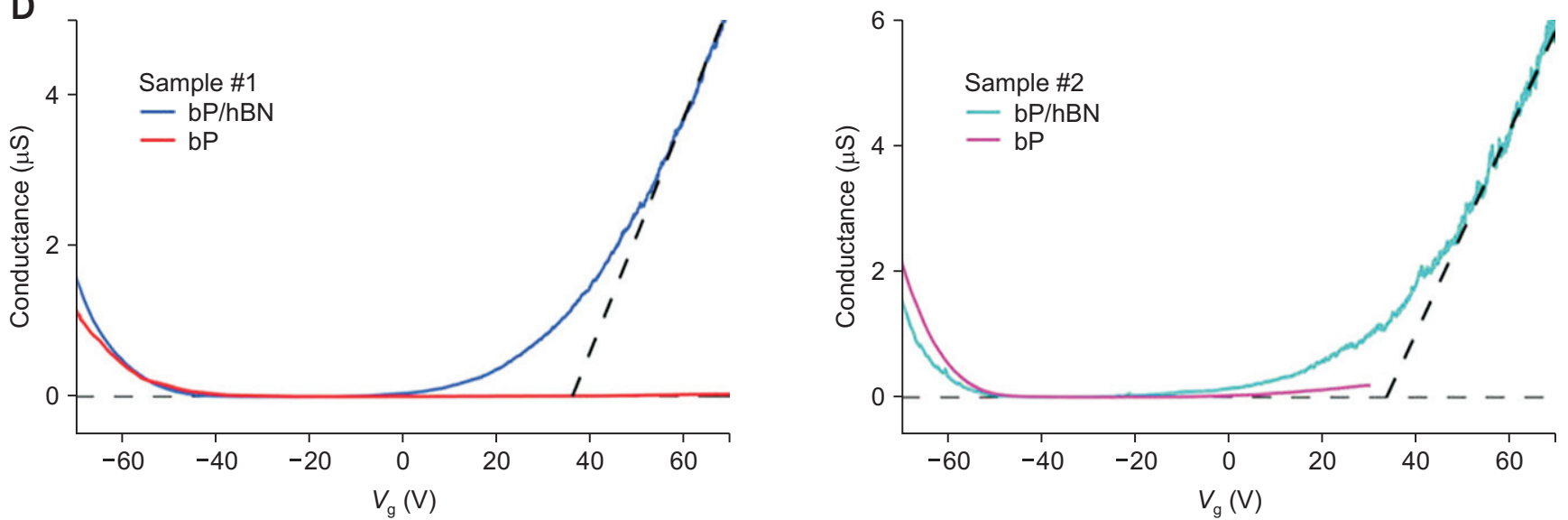

Fig. 9. (A) Raman spectrum of passivated area of black phosphorus (BP) flakes showing BP peaks and graphene peak (inset). (B) Schematic threedimensional illustration of device geometry. (C, D) Conductance versus back-gate voltage (S- $\mathrm{V}_{\mathrm{g}}$ ) of passivated and unpassivated channels of two BP fieldeffect transistor (FET) devices at $\mathrm{V}_{\mathrm{SD}}=50 \mathrm{mV}$ and $\mathrm{T}=300 \mathrm{~K}$. The thickness of $\mathrm{BP}$ is $\sim 4.5 \mathrm{~nm}$ and $\sim 5.7 \mathrm{~nm}$ for sample \#1 and \#2, respectively. Reprinted from Doganov et al. (2015) (Nat. Commun. 6, 6647), with permission from Nature.

To determine out the performance of the transferred graphene or few-layer hBN in passivating the BP flake, AFM and Raman spectroscopy were performed.

Fig. $8 \mathrm{~A}$ and B show AFM morphologies of the $\sim 5 \mathrm{~nm}$ thick pristine BP flakes covered partially with graphene and exposed to ambient conditions for $10 \mathrm{~min}$ and $24 \mathrm{~h}$, respectively. From the images, it is obvious that the surface of the unpassivated part of the BP flakes is very rough, while the passivated areas of the BP flakes do not show any noticeable change in roughness. Fig. $8 \mathrm{C}$ shows the AFM morphology of the $10 \mathrm{~nm}$-thick pristine BP flake covered partially with $\mathrm{hBN}$, and it shows similar performance as graphene. Similarly, Fig. 8C-E, which show the average roughness configurations according to the exposure times of the unpassivated and the passivated BP flakes, are similar to Fig. 8A-C.

Moreover, the Raman spectra of the passivation materials do not show any variation during the exposure time and are not modified by BP flakes, which indicates the presence of physical bonds (van der Waals interactions) between the passivating layers and the BP flake (Fig. 9A). Therefore, by preventing the BP flakes from being invaded by using oxygen and water by $2 \mathrm{D}$ materials (graphene and $\mathrm{hBN}$ ), Doganov et al. (2015) succeeded in preventing charge transfer between phosphorus atom and aqueous oxygen.

According to Fig. 9C, in both types of unpassivated BP (red line-top, purple line-bottom), conductance versus backgate voltage shows that the pristine BP exhibits p-type semiconducting property, meaning that conductance corresponding to electrons is almost 0 , and conductance corresponding to hole carriers is about $2 \mu \mathrm{S}$ at $\mathrm{V}_{\mathrm{g}}=-70 \mathrm{~V}$.

By contrast, in case of the passivated hBN/BP crystal (blue line-top, green line-bottom), the conductance versus backgate voltage curve shows a considerable increase in electron conductance $\left(\mathrm{V}_{\mathrm{g}}>0\right)$, which is much higher than hole conductance $\left(\mathrm{V}_{\mathrm{g}}<0\right)$; thus, the $\mathrm{hBN}$-passiavted BP ET device exhibits symmetric electron and hole transconductance. 
Given that carrier mobility is proportional to the derivative of conductance with respect to gate voltage $\left(\mathrm{dC} / \mathrm{dV}_{\mathrm{g}}\right)$, it is evident that the passivation affects the mobility of both carriers as well.

From the conductance versus back-gate voltage plot above, which shows the transconductance of the unoxidized BP FET, Doganov et al. (2015) stated that the p-type transport property of pristine $\mathrm{BP}$ can be ascribed to the trapping of electron carriers by oxygen point defects and oxygen/water redox couple, while hole carriers are not trapped. This means charge transfer occurs between phosphorus and aqueous oxygen, which leads to p-type doping of BP.

\section{CONCLUSIONS}

In summary, types of passivation methods for BP flakes were reviewed, such as chemical passivation along with functionalization of BP surface with aryl diazonium salt solutions (4-NBD, 4-MBD) by forming strong P-C bonds, formation of stable aluminum oxide $\left(\mathrm{AlO}_{\mathrm{x}}\right)$ layers using $\mathrm{ALD}$, and, the physical passivation through direct transfer with highly-mechanically stable 2D materials (graphene, hBN) onto the surface of BP. All methods show reliable passivation performance in terms of preventing the degradation of $\mathrm{BP}$, and they modify the electronic properties of BP, uniquely.

There exist only a few methods for the passivation of BP flake, and except ALD passivation, all other methods employ wet processes, for example immersing BP flakes into aryl diazonium salt solutions for seconds and removing the PMMA polymer by using acetone, which has negative effects on fabricating or running a device. In addition, in case of the direct transfer method using graphene or hBN for a passivation layer, they cannot encapsulate the underlying BP flakes perfectly owing to the interface, defects innate in the crystals, suggesting the possibility of aqueous oxygen invading through graphene/BP (or hBN/BP) interface. This brings into question the long-term reliability of the method compared to other methods that cancel the possibility of charge transfer at the fundamental level.

Since few-layers BP is strongly reactive to oxygen and water, with a relatively low thermal decomposition temperature (Liu et al., 2015), it is not easy to apply existing passivation methods. Moreover, most of these methods do not yield reliable performance and do not ensure that BP flakes do not degrade in the long term. Therefore, it is required to discover methods that forms strong phosphoric bonds with passivation materials, which ensures long-term reliability without any processes that hinder the unique properties of BP.

\section{CONFLICT OF INTEREST}

No potential conflict of interest relevant to this article was reported.

\section{ACKNOWLEDGMENTS}

This work was supported by the National Research Foundation of Korea (NRF) grant funded by the Korea government (MSIP) (No. 2015R1A2A2A01006992) and Nano Material Technology Development Program (2012M3A7B4049807).

\section{REFERENCES}

Doganov R A, O'Farrell E C T, Koenig S P, Yeo Y T, Ziletti A, Carvalho A, Campbell D K, Coker D F, Watanabe K, Taniguchi T, Neto A H C, and Ozyilmaz B (2015) Transport properties of pristine fewlayer black phosphorus by van der Waals passivation in an inert atmosphere. Nat. Commun. 6, 6647.

Du H W, Lin X, Xu Z M, and Chu D W (2015) Recent developments in black phosphorus transistors. J. Mater. Chem. C 3, 8760-8775.

Favron A, Gaufres E, Fossard F, Phaneuf-L'Heureux A L, Tang N Y W, Levesque P L, Loiseau A, Leonelli R, Francoeur S, and Martel R (2015) Photooxidation and quantum confinement effects in exfoliated black phosphorus. Nat. Mater. 14, 826-833.

Geim A K and Novoselov K S (2007) The rise of graphene. Nat. Mater. 6 , 183-191.

Kim J, Baik S S, Ryu S H, Sohn Y, Park S, Park B G, Denlinger J, Yi Y, Choi H J, and Kim K S (2015) Observation of tunable band gap and anisotropic Dirac semimetal state in black phosphorus. Science 349, 723-726.
Lee S Y, Duong D L, Vu Q A, Jin Y, Kim P, and Lee Y H (2015) Chemically modulated band gap in bilayer graphene memory transistors with high on/off ratio. Acs Nano. 9, 9034-9042.

Liu X L, Wood J D, Chen K S, Cho E, and Hersam M C (2015) In situ thermal decomposition of exfoliated two-dimensional black phosphorus. J. Phys. Chem. Lett. 6, 773-778.

Novoselov K S, Geim A K, Morozov S V, Jiang D, Katsnelson M I, Grigorieva I V, Dubonos S V, and Firsov A A (2005) Two-dimensional gas of massless Dirac fermions in graphene. Nature 438, 197-200.

Piao Y M, Meany B, Powell L R, Valley N, Kwon H, Schatz G C, and Wang Y H (2013) Brightening of carbon nanotube photoluminescence through the incorporation of $\mathrm{sp}(3)$ defects. Nat. Chem. 5, 840-845.

Ritala M, Kukli K, Rahtu A, Raisanen P I, Leskela M, Sajavaara T, and Keinonen J (2000) Atomic layer deposition of oxide thin films with metal alkoxides as oxygen sources. Science 288, 319-321.

Ryder C R, Wood J D, Wells S A, Yang Y, Jariwala D, Marks T J, Schatz G C, and Hersam M C (2016) Covalent functionalization and passivation 
of exfoliated black phosphorus via aryl diazonium chemistry. Nat. Chem. 8, 597-602.

Seo S, Lee H U, Lee S C, Kim Y, Kim H, Bang J, Won J, Kim Y, Park B, and Lee J (2016) Triangular black phosphorus atomic layers by liquid exfoliation. Sci. Rep. 6, 23736.

Sugai S and Shirotani I (1985) Raman and infrared reflection spectroscopy in black phosphorus. Solid State Commun. 53, 753-755.

Tan J Y, Avsar A, Balakrishnan J, Koon G K W, Taychatanapat T, O'Farrell E C T, Watanabe K, Taniguchi T, Eda G, Neto A H C, and Ozyilmaz B
(2014) Electronic transport in graphene-based heterostructures. Appl. Phys. Lett. 104, 183504.

Watanabe K, Taniguchi T, and Kanda H (2004) Direct-bandgap properties and evidence for ultraviolet lasing of hexagonal boron nitride single crystal. Nat. Mater. 3, 404-409.

Wood J D, Wells S A, Jariwala D, Chen K S, Cho E, Sangwan V K, Liu X L, Lauhon L J, Marks T J, and Hersam M C (2014) Effective passivation of exfoliated black phosphorus transistors against ambient degradation. Nano Lett. 14, 6964-6970. 